\title{
Five extracellular matrix-associated genes upregulated in oral tongue squamous cell carcinoma: An integrated bioinformatics analysis
}

\author{
PINGPING ZHONG ${ }^{1-4^{*}}$, LIYA LIU ${ }^{5,6^{*}}$, ALING SHEN $^{5,6}$, ZHONGXIN CHEN $^{7}$, XIAOYAN HU $^{2-4}$, \\ YICHAO CAI ${ }^{2-4}, \mathrm{JIE} \mathrm{LIN}^{2-4}$, BANGYAN WANG $^{2-4}$, JIESEN LI ${ }^{2-4}$, YOUQIN CHEN ${ }^{8}$ and JUN PENG ${ }^{5,6}$ \\ ${ }^{1}$ Department of Orthodontics, School and Hospital of Stomatology, Fujian Medical University; ${ }^{2}$ Key Laboratory of \\ Stomatology, Fujian Province University; ${ }^{3}$ Fujian Biological Materials Engineering and Technology Center of Stomatology; \\ ${ }^{4}$ Institute of Stomatology, Fujian Medical University, Fuzhou, Fujian 350002; ${ }^{5}$ Academy of Integrative Medicine; \\ ${ }^{6}$ Fujian Key Laboratory of Integrative Medicine on Geriatrics, Fujian University of Traditional Chinese Medicine, \\ Fuzhou, Fujian 350122; ${ }^{7}$ West China School of Stomatology Sichuan University, Chengdu, \\ Sichuan 610041, P.R. China; ${ }^{8}$ Department of Pediatrics, Case Western Reserve University School \\ of Medicine, Rainbow Babies and Children's Hospital, Cleveland, OH 44106, USA
}

Received November 3, 2018; Accepted July 26, 2019

DOI: $10.3892 / \mathrm{ol} .2019 .10982$

\begin{abstract}
Despite advancements in treatment regimens, the mortality rate of patients with oral tongue squamous cell carcinoma (OTSCC) is high. In addition, the signaling pathways and oncoproteins involved in OTSCC progression remain largely unknown. Therefore, the aim of the present study was to identify specific prognostic marker for patients at a high risk of developing OTSCC. The present study used four original microarray datasets to identify the key candidate genes involved in OTSCC pathogenesis. Expression profiles of 93 OTSCC tissues and 76 normal tissues from GSE9844, GSE13601, GSE31056
\end{abstract}

Correspondence to: Professor Youqin Chen, Department of Pediatrics, Case Western Reserve University School of Medicine, Rainbow Babies and Children's Hospital, 11100 Euclid Avenue, Cleveland, OH 44106, USA

E-mail: yxc571@case.edu

Professor Dr Jun Peng, Fujian Key Laboratory of Integrative Medicine on Geriatrics, Fujian University of Traditional Chinese Medicine, 1 Huatuo Road, Minhou Shangjie, Fuzhou, Fujian 350122, P.R. China

E-mail: pjunlab@hotmail.com

${ }^{*}$ Contributed equally

Abbreviations: HNSC, head and neck squamous cell carcinoma; OTSCC, oral tongue squamous cell carcinoma; DEG, differentially expressed gene; TCGA, The Cancer Genome Atlas; GEO, Gene Expression Omnibus; GO, Gene Ontology

Key words: head and neck squamous cell carcinoma, oral tongue squamous cell carcinoma, bioinformatics analysis, differentially expressed gene and GSE75538 datasets were investigated. Differentially expressed genes (DEGs) were determined, and gene ontology enrichment and gene interactions were analyzed. The four GSE datasets reported five upregulated and six downregulated DEGs. Five upregulated genes (matrix metalloproteinase 1, 3, 10 and 12 and laminin subunit gamma 2) were localized in the extracellular region of cells and were associated with extracellular matrix disassembly. Furthermore, analysis for The Cancer Genome Atlas database revealed that the aforementioned five upregulated genes were also highly expressed in OTSCC and head and neck squamous cell carcinoma tissues. These results demonstrated that the five upregulated genes may be considered as potential prognostic biomarkers of OTSCC and may serve at understanding OTSCC progression. Upregulated DEGs may therefore represent valuable therapeutic targets to prevent or control OTSCC pathogenesis.

\section{Introduction}

Head and neck squamous cell carcinoma (HNSC) is a common cancer worldwide and accounts for $>600,000$ new cases annually (1). Despite significant advancements in treatments, including reconstructive microvascular free tissue transfer, hyperfractionated radiotherapy and concurrent chemoradiation, the survival rate of patients with HNSC has not sufficiently improved over the last 50 years, with overall survival of $\sim 50 \%$, resulting in increased mortality rates worldwide every year (2). HNSC is a highly complex disease emerging from the oral cavity, tongue, pharynx or larynx (3). Each tumor harbors unique mutations, presents variable clinical outcomes and is associated with specific risk factors $(4,5)$. For example, TP53 inactivation, either through somatic mutation or HPV infection, appears nearly universal in this malignancy (6). The present study particularly focused on one type of HNSC, the oral tongue squamous cell carcinoma (OTSCC), because of its poor diagnosis, high incidence 
rate, aggressive clinical behavior and poor outcome (7-10). A recent study reported a five-year survival rate of $63 \%$ for patients with OTSCC in The Netherlands (11). In 2017, nearly 16,400 new cases of tongue cancer were diagnosed and 2,400 tongue cancer-associated mortality cases were recorded in the United States (12). Several prognostic factors for OTSCC exist, including occult node positivity, tumor depth, lymphovascular invasion and perineural invasion (13). Nevertheless, robust and reliable molecular prognostic biomarkers need to be determined in order to identify patients with advanced stages of OTSCC.

Several thousands of tumor biomarkers have been discovered and are associated with the prognosis of various types of cancer (14). In particular, markers for OTSCC, including microtubule associated scaffold protein 1 and beta-parvin, have attracted much attention due to their crucial role in OTSCC pathogenesis (15). Furthermore, it was demonstrated that downregulation of these markers markedly decreases cancer cell survival (15-19).

Thanks to the rapid development and extensive application of microarrays for gene identification in various types of cancer (20-22), a high number of differentially expressed genes (DEGs) in OTSCC have been identified $(13,19)$. However, the results between studies are inconsistent, which might be due the variability of tissue samples used. In addition, no reliable biomarkers for OTSCC have been established. Subsequently, the combination of bioinformatics methods and expression profiling techniques may represent a novel approach to resolve these problems. The present study used four microarray datasets [GSE9844 (3), GSE13601 (23), GSE31056 (24), and GSE75538 (25)] from the National Center for Biotechnology Information (NCBI)-Gene Expression Omnibus (GEO) database and an mRNA sequencing (mRNA-seq) dataset from The Cancer Genome Atlas (TCGA). DEGs were filtered using the GEO2R tool according to conventional data processing standards, and gene ontology (GO) enrichment analysis was performed to screen for DEGs using The Database for Annotation, Visualization and Integrated Discovery (DAVID). Expression levels of DEGs in samples from TCGA were assessed using Gene Expression Profiling Interactive Analysis (GEPIA), and corrections were applied for DEGs is samples from TCGA-HNSC using cBioPortal to identify potential oncogenes in OTSCC. The identified DEGs and their associated pathways may be considered as robust and reliable tumor biomarkers for OTSCC and serve as precise therapeutic targets for the prevention of OTSCC progression at early stages.

\section{Materials and methods}

GEO datasets. GEO (https://www.ncbi.nlm.nih.gov/gds) is a public repository at NCBI for storing high-throughput gene expression data (26). The gene expression profiles of GSE9844, GSE13601, GSE31056, and GSE75538 were selected from the GEO database (http://www.ncbi.nlm.nih.gov/geo/). GSE9844 included 26 OTSCC and 12 normal tissues, GSE13601 included 31 OTSCC and 26 normal tissues, GSE31056 included 22 OTSCC and 24 normal tissues, and GSE75538 included 14 OTSCC and 14 normal tissues.

Identification of DEGs. GEO2R (https://www.ncbi.nlm.nih. gov/geo/geo2r/) was used to detect DEGs between OTSCC and
Table I. Characteristics of 147 patients with tongue tumor from The Cancer Genome Atlas database.

\begin{tabular}{|c|c|c|}
\hline Characteristics & Patient number & Percentage \\
\hline \multicolumn{3}{|l|}{ Sex } \\
\hline Female & 46 & 31.29 \\
\hline Male & 101 & 68.71 \\
\hline \multicolumn{3}{|c|}{ Age at diagnosis, years } \\
\hline Mean & $58.60 \pm 12.71$ & \\
\hline$<40$ & 10 & 6.80 \\
\hline $40-49$ & 20 & 13.61 \\
\hline $50-59$ & 38 & 25.85 \\
\hline $60-69$ & 53 & 36.05 \\
\hline $70-79$ & 21 & 14.29 \\
\hline$>80$ & 4 & 2.72 \\
\hline \multicolumn{3}{|c|}{ Tumor pathological stage } \\
\hline $\mathrm{T} 1-\mathrm{T} 2$ & 70 & 47.62 \\
\hline T3-T4 & 72 & 48.98 \\
\hline \multicolumn{3}{|c|}{ Nodal pathological stage } \\
\hline N0-N1 & 72 & 48.98 \\
\hline $\mathrm{N} 2-\mathrm{N} 4$ & 70 & 47.62 \\
\hline \multicolumn{3}{|l|}{ Smoking history } \\
\hline Yes & 98 & 66.67 \\
\hline No/Never & 47 & 31.97 \\
\hline Unknown & 2 & 1.36 \\
\hline \multicolumn{3}{|l|}{ Alcohol history } \\
\hline Yes & 99 & 67.35 \\
\hline No/Never & 44 & 29.93 \\
\hline Unknown & 4 & 2.72 \\
\hline \multicolumn{3}{|c|}{ Primary lymph node } \\
\hline Yes & 128 & 87.07 \\
\hline No & 9 & 6.12 \\
\hline
\end{tabular}

normal tissue samples (27). Adjusted P-values $<0.01$ and Ilog fold change $(\mathrm{FC}) \mid>2$ were set as cutoffs. Co-expressed DEGs that were downregulated or upregulated in the two sets of gene expression profiles were identified using Venn diagrams (http://bioinformatics.psb.ugent.be/webtools/Venn/).

GO enrichment analysis of DEGs. Usually, genes and their products are annotated to identify characteristic biological function and processes of a high-throughput genome or transcriptome (28). DAVID (https://david.ncifcrf.gov/) is a web-based bioinformatics resource for gene annotation and visualization with an integrated discovery function. It is therefore useful for determining gene biological attributes (29). $\mathrm{P}<0.01$ and the Benjamini corrected $\mathrm{P}<0.01$ (30) were set as the cutoff. Sequential pathways, molecular and cellular components and biological functions of DEGs could be visualized by using DAVID (https://david.ncifcrf.gov/).

Comparison of gene expression in patients with OTSCC. The mRNA-seq data of the five OTSCC genes of interest from HNSC samples were obtained from the TCGA database 
Table II. Gene ontology analysis of upregulated genes associated with oral tongue squamous cell carcinoma.

\begin{tabular}{|c|c|c|c|c|c|}
\hline Category & Term & Count & Genes & P-value & $\begin{array}{c}\text { Benjamini } \\
\text { corrected } \\
\text { P-value }\end{array}$ \\
\hline GOTERM_BP_DIRECT & $\begin{array}{l}\text { GO:0022617 extracellular } \\
\text { matrix disassembly }\end{array}$ & 5 & $\begin{array}{l}\text { MMP10, LAMC2, MMP3, } \\
\text { MMP12, MMP1 }\end{array}$ & $3.9 \times 10^{-10}$ & $7.7 \times 10^{-9}$ \\
\hline GOTERM_BP_DIRECT & $\begin{array}{l}\text { GO:0030574 collagen } \\
\text { catabolic process }\end{array}$ & 4 & MMP10, MMP3, MMP12, MMP1 & $2.1 \times 10^{-7}$ & $2.1 \times 10^{-6}$ \\
\hline GOTERM_MF_DIRECT & $\begin{array}{l}\text { GO:0004222 } \\
\sim \text { metalloendopeptidase } \\
\text { activity }\end{array}$ & 4 & MMP10, MMP3, MMP12, MMP1 & $1.2 \times 10^{-6}$ & $9.3 \times 10^{-6}$ \\
\hline GOTERM_CC_DIRECT & $\begin{array}{l}\text { GO:0005578 proteinaceous } \\
\text { extracellular matrix }\end{array}$ & 4 & MMP10, MMP3, MMP12, MMP1 & $1.2 \times 10^{-5}$ & $1.1 \times 10^{-4}$ \\
\hline GOTERM_MF_DIRECT & $\begin{array}{l}\text { GO:0004252 serine-type } \\
\text { endopeptidase activity }\end{array}$ & 4 & MMP10, MMP3, MMP12, MMP1 & $1.3 \times 10^{-5}$ & $5.4 \times 10^{-5}$ \\
\hline GOTERM_MF_DIRECT & $\begin{array}{l}\text { GO:0004175 endopeptidase } \\
\text { activity }\end{array}$ & 3 & MMP3, MMP12, MMP1 & $6.0 \times 10^{-5}$ & $1.6 \times 10^{-4}$ \\
\hline GOTERM_CC_DIRECT & $\begin{array}{l}\text { GO:0005576 extracellular } \\
\text { region }\end{array}$ & 5 & $\begin{array}{l}\text { MMP10, LAMC2, MMP3, MMP12, } \\
\text { MMP1 }\end{array}$ & $6.1 \times 10^{-5}$ & $2.7 \times 10^{-4}$ \\
\hline GOTERM_BP_DIRECT & GO:0006508 proteolysis & 4 & MMP10, MMP3, MMP12, MMP1 & $1.0 \times 10^{-4}$ & $6.8 \times 10^{-4}$ \\
\hline GOTERM_MF_DIRECT & $\begin{array}{l}\text { GO:0005509 calcium ion } \\
\text { binding }\end{array}$ & 4 & MMP10, MMP3, MMP12, MMP1 & $3.0 \times 10^{-4}$ & $5.9 \times 10^{-4}$ \\
\hline GOTERM_MF_DIRECT & GO:0008270 zinc ion binding & 4 & MMP10, MMP3, MMP12, MMP1 & $1.3 \times 10^{-3}$ & $2.0 \times 10^{-3}$ \\
\hline
\end{tabular}

(https://cancergenome.nih.gov/) (31). In addition, clinical data of OTSCC were downloaded using TCGA Assembler (Table I). mRNA-seq data of 147 OTSCC tissues and 15 adjacent normal tongue tissues were obtained on an Illumina HiSeq RNASeq platform (32). Since the TCGA dataset was developed as a community resource project, no additional approval was required for this study from Fujian Medical University. The present study complied with the TCGA publication guidelines and data access policies (33).

Analysis of gene expression patterns in all tumors from TCGA through GEPIA. The expression patterns of the five genes of interest in various types of cancers and normal tissues were determined using GEPIA (http://gepia.cancer-pku.cn) (34). The program was used to analyze RNA sequencing data of 33 types of cancer (including adrenocortical carcinoma, bladder urothelial carcinoma, breast invasive carcinoma (BRCA), cervical squamous cell carcinoma and endocervical adenocarcinoma (CESC), cholangiocarcinoma (CHOL), colon adenocarcinoma (COAD), lymphoid neoplasm diffuse large B-cell lymphoma, esophageal carcinoma (ESCA), glioblastoma multiforme, head and neck squamous cell carcinoma (HNSC), kidney chromophobe (KICH), kidney renal clear cell carcinoma, kidney renal papillary cell carcinoma, acute myeloid leukemia, brain lower grade glioma, liver hepatocellular carcinoma, lung adenocarcinoma (LUAD), lung squamous cell carcinoma (LUSC), mesothelioma, ovarian serous cystadenocarcinoma, pancreatic adenocarcinoma, pheochromocytoma and paraganglioma, prostate adenocarcinoma, rectum adenocarcinoma (READ), sarcoma, skin cutaneous melanoma, stomach adenocarcinoma (STAD), testicular germ cell tumor, thyroid carcinoma, thymoma, uterine corpus endometrial carcinoma, uterine carcinosarcoma, uveal melanoma) and adjacent normal samples from TCGA following the standard processing pipeline. P-value $<0.01$ and $\mid \log \mathrm{FCl}>2$ were set as cutoffs. Box plots were generated to visualize the associations.

Correlations among genes expression in HNSC samples from TCGA through cBioPortal. The correlations between expression patterns of the genes of interest in cancer tissues from TCGA-HNSC dataset were analyzed using Pearson and Spearman correlation coefficient via cBioPortal (http://www. cbioportal.org) (35-36).

Statistical analysis. Statistical analyses were performed using Graphpad Prism 5.0 (GraphPad Software, Inc.). Mann-Whitney $\mathrm{U}$ test was performed to compare gene expression between tumor and adjacent normal tissues. A two-tailed P-value $<0.01$ was considered statistically significant.

\section{Results}

Some biomarkers distinguished OTSCC tissues from normal tissues. Four GEO datasets (GSE9844, GSE13601, GSE31056, and GSE75538) were imported into the GEO2R analysis tool. Based on DEGs between OTSCC and normal tissues, five upregulated genes [matrix metalloproteinases 1, 3, 10 and 12 (MMP1, MMP3, MMP10 and MMP12) and laminin subunit gamma 2 (LAMC2)] (Fig. 1A) and six downregulated genes (dermatopontin, cartilage intermediate layer protein, keratin 4 , ATP binding cassette subfamily A member 8 , alcohol dehydrogenase $1 \mathrm{~B}$ and protein phosphatase 1 regulatory 

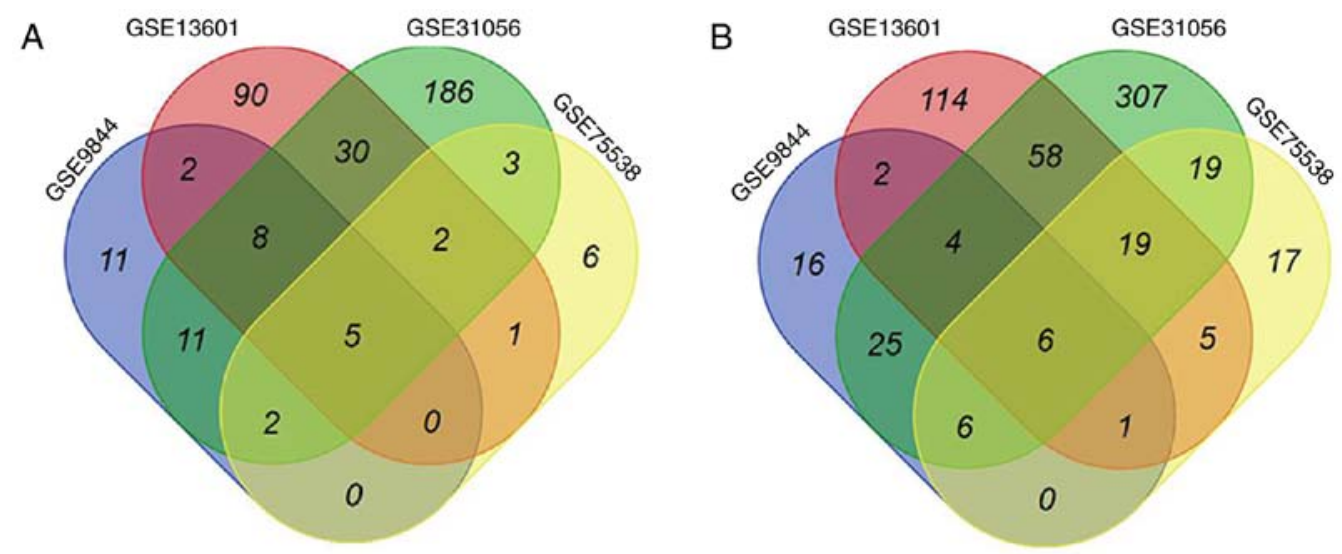

Figure 1. Venn diagram of (A) upregulated and (B) downregulated DEGs in four GEO datasets (GSE9844, GSE13601, GSE31056 and GSE75538). Each colored area represents the number of DEGs in one corresponding dataset. The cross-areas indicated altered DEGs. DEGs were identified using Mann-Whitney $U$ test and statistically significant DEGs were defined for $\mathrm{P}<0.01$ and (A) $\log \mathrm{FC}>2$ (A) or (B) $\log \mathrm{FC}<-2$ as cutoffs. DEGs, differentially expressed genes.
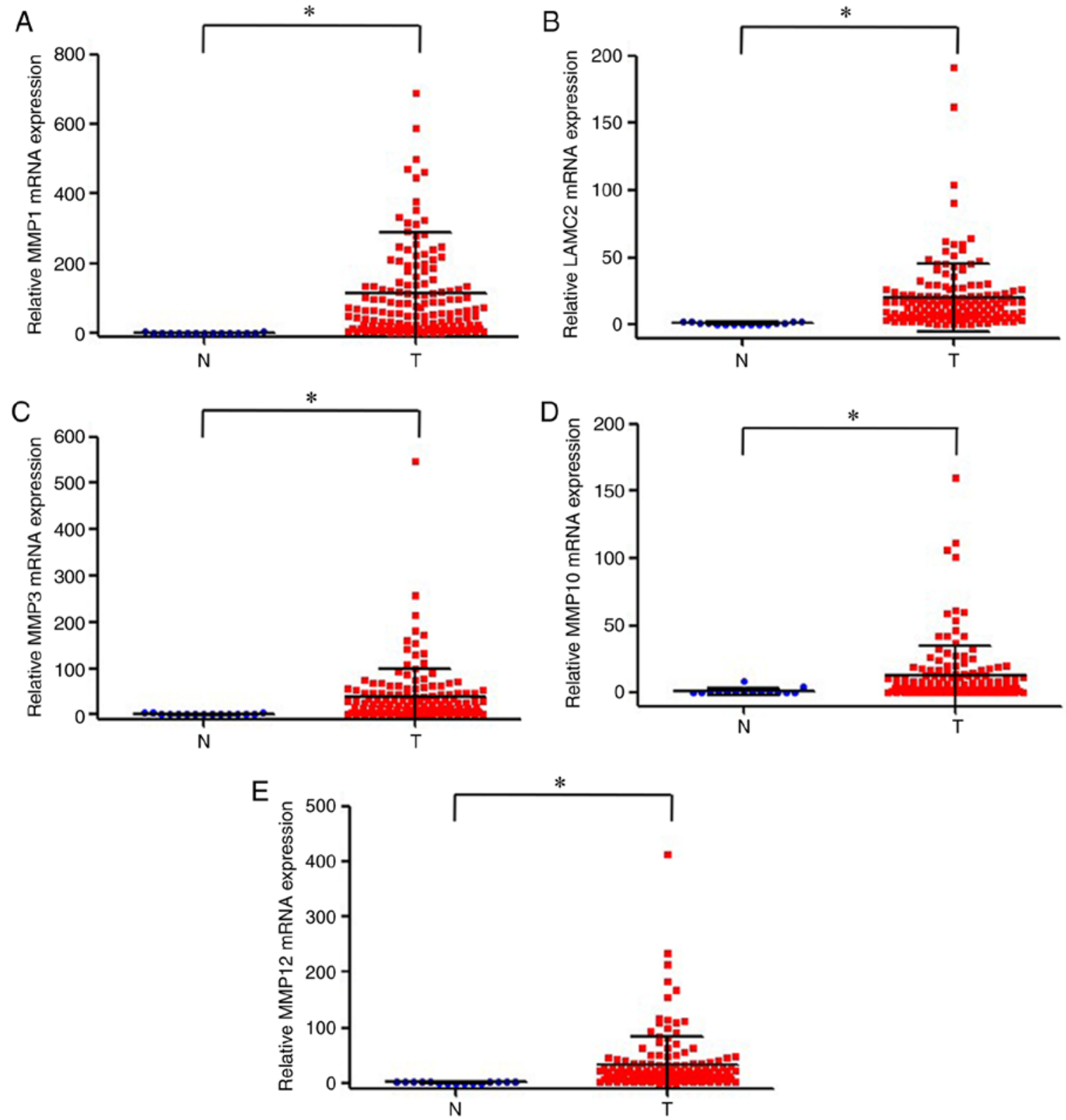

Figure 2. Expression levels of (A) $M M P 1$, (B) $L A M C 2$, (C) $M M P 3$, (D) $M M P 10$ and (E) $M M P 12$ in oral tongue squamous cell carcinoma and normal tissues based on TCGA database. "P<0.01. LAMC2, laminin subunit gamma 2; MMP1, 3, 10 and 12, matrix metalloproteinase 1, 3, 10 and 12; N, normal tissue; $\mathrm{T}$, cancer tissue. 
A
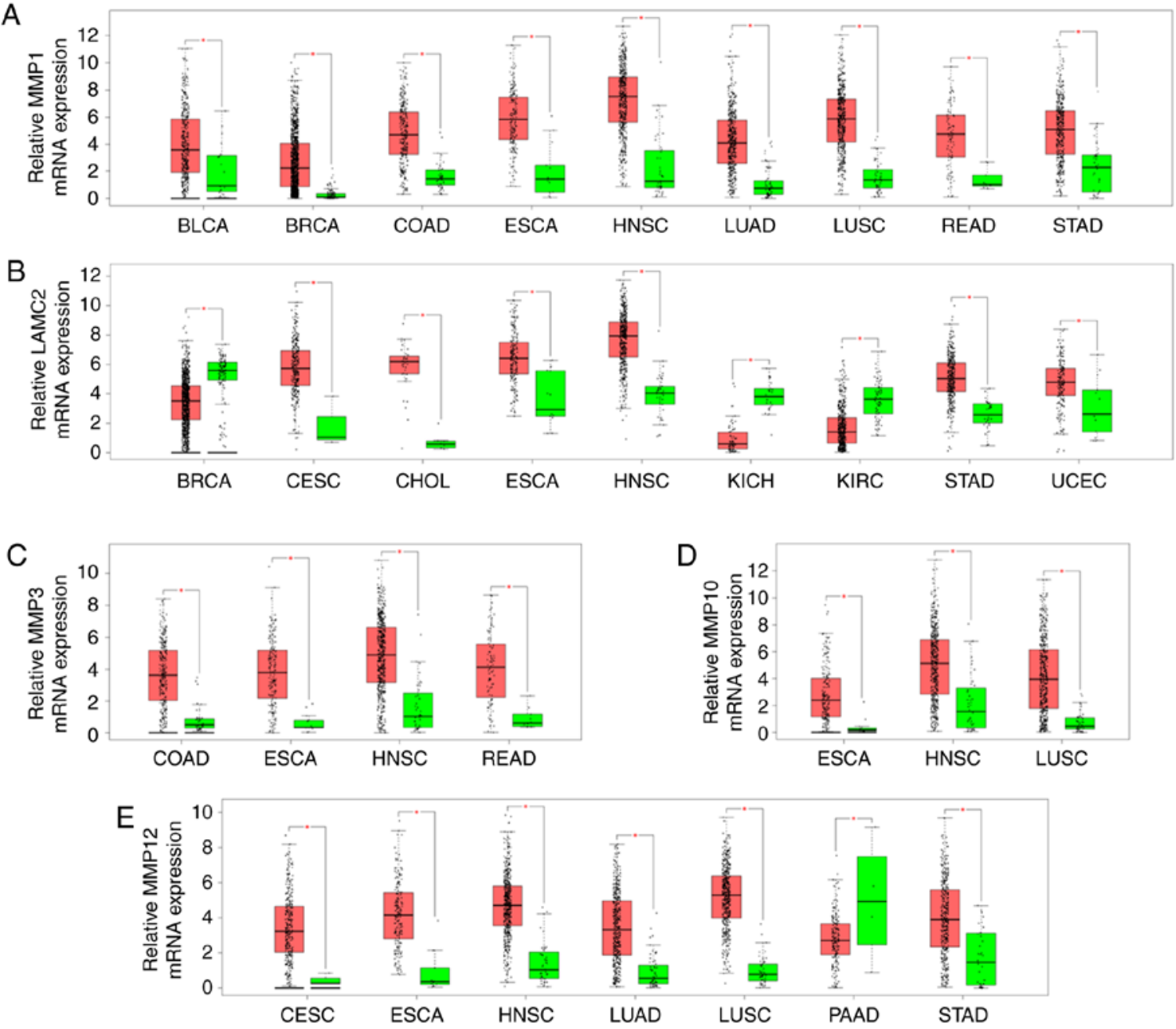

Figure 3. Comparison of $M M P 1, L A M C 2, M M P 3, M M P 10$ and $M M P 12$ expressions between cancer (Red) and normal tissues (Green) among 23 various types of cancer from The Cancer Genome Atlas according to GEPIA program. (A) MMP1; (B) LAMC2; (C) $M M P 3$; (D) $M M P 10$; (E) MMP12. The Y axis indicates the $\log 2(\mathrm{TPM}+1)$ for gene expression. The red and the green bars represent the cancer and normal tissues, respectively. These figures were derived from GEPIA. ${ }^{*} \mathrm{P}<0.01$. BLCA, bladder urothelial carcinoma; BRCA, breast invasive carcinoma; CESC, cervical squamous cell carcinoma and endocervical adenocarcinoma; CHOL, cholangiocardinoma; COAD, colon adenocarcinoma; ESCA, esophageal carcinoma; GEPIA, Gene Expression Profiling Interactive Analysis; HNSC, head and neck squamous cell carcinoma; KICH, kidney chromophobe; KIRC, kidney renal clear cell carcinoma; LAMC2, laminin subunit gamma 2; LUAD, lung adenocarcinoma; LUSC, lung squamous cell carcinoma; MMP1, 3, 10 and 12, matrix metalloproteinase 1, 3, 10 and 12; PAAD, pancreatic adenocarcinoma; READ, rectum adenocarcinoma; STAD, stomach adenocarcinoma; UCEC, uterine corpus endometrioid carcinoma; TPM, transcripts per million.

subunit 3C) (Fig. 1B) were highlighted in all four datasets. Whether the five upregulated genes could be considered as potential biomarkers for distinguishing OTSCC tissues from normal tissues was therefore assessed.

GO function enrichment analysis. GO enrichment analysis was performed using DAVID tool. The five specific upregulated genes MMP1, LAMC2, MMP3, MMP10 and MMP12 were uploaded into the DAVID software. GO results indicated that these genes were specifically involved in certain biological processes, including 'extracellular matrix disassembly', 'collagen catabolism' and 'proteolysis'. Furthermore, with regards to the molecular function, the products of these five genes mainly comprised metalloendopeptidases, serine-type endopeptidases, calcium ion binders and zinc ion binders. Similarly, GO cell component analysis revealed that the proteins encoded by these genes were significantly enriched for functions associated with the proteinaceous extracellular matrix and extracellular region $(\mathrm{P}<0.01$, Table II).
Gene expression of MMP1, LAMC2, MMP3, MMP10 and MMP12 in OTSCC samples from TCGA. TCGA database was used to analyze the expression levels of the five genes of interest in OTSCC and adjacent normal tissues. The results demonstrated that the expression levels of MMP1, LAMC2, $M M P 3, M M P 10$ and $M M P 12$ were significantly upregulated in OTSCC tissues compared with adjacent normal tissues (Fig. 2).

Gene expression of MMP1, LAMC2, MMP3, MMP10 and MMP12 in 23 cancer samples from TCGA. Consistent results were obtained for bladder urothelial carcinoma, BRCA, COAD, ESCA, HNSC, LUAD, LUSC, READ and STAD. MMP1 expression level was significantly increased in all cancer tissues compared with adjacent normal tissues (Fig. 3A). LAMC2 was upregulated in CESC, CHOL, ESCA, HNSC, STAD and uterine corpus endometrial carcinoma, whereas it was significantly downregulated in BRCA, kidney chromophobe and kidney renal clear cell carcinoma (Fig. 3B). Furthermore, MMP3 expression was upregulated in COAD, 

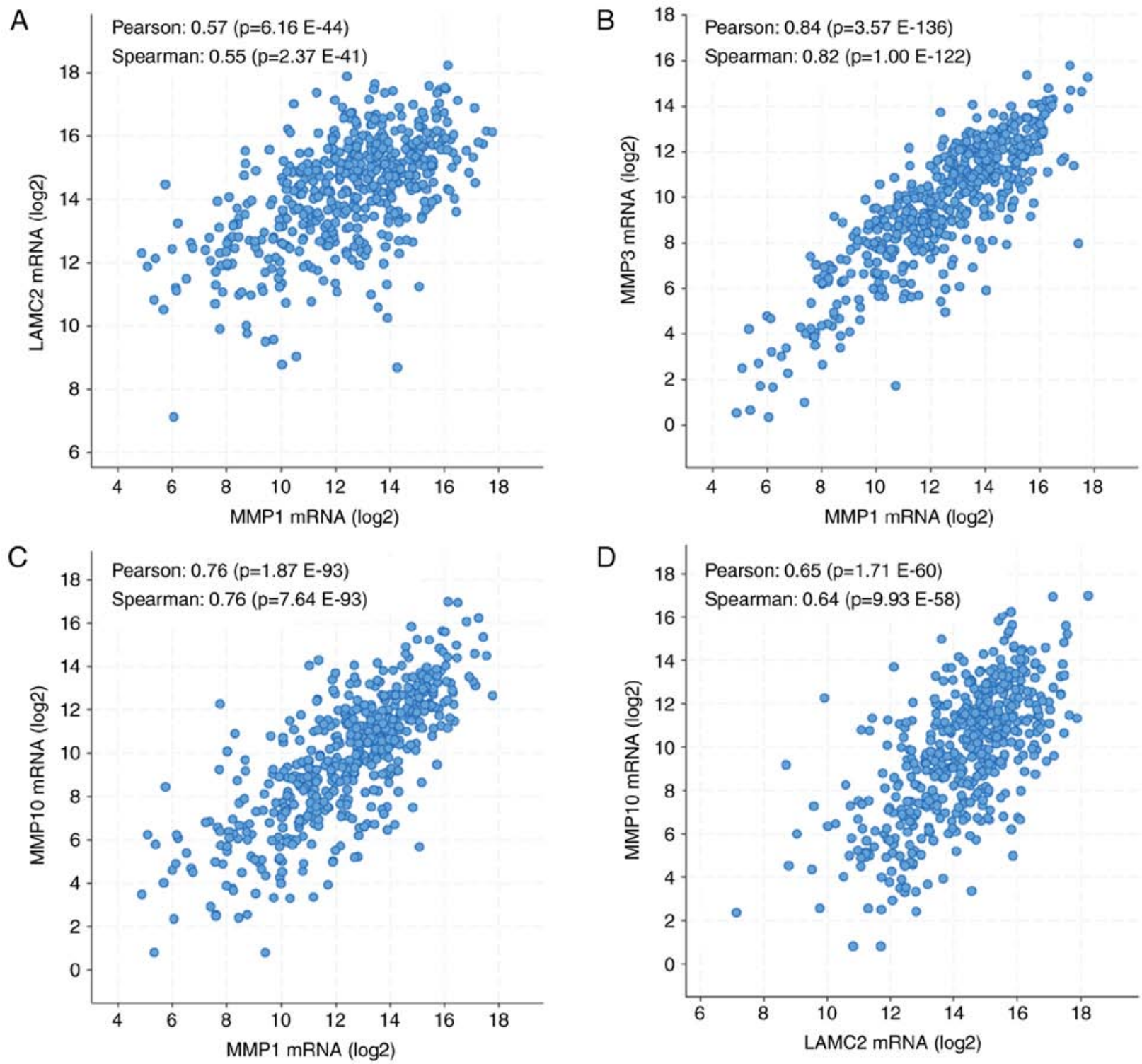

Figure 4. Correlations between $M M P 1, L A M C 2, M M P 3, M M P 10$ and $M M P 12$ expression levels in HNSC tissues. (A) Correlation between $M M P 1$ and $L A M C 2$ expression levels in HNSC tissues. (B) Correlation between $M M P 1$ and $M M P 3$ expression levels in HNSC tissues. (C) Correlation between MMP1 and $M M P 10$ expression levels in HNSC tissues. (D) Correlation between LAMC2 and MMP10 expression levels in HNSC tissues. Analyses were performed using cBioPortal on the provisional datasets from The Cancer Genome Atlas. HNSC, head and neck squamous cell carcinoma; LAMC2, laminin subunit gamma 2; MMP1, 3, 10 and 12, matrix metalloproteinase 1,3,10 and 12 .

ESCA, HNSC, and READ (Fig. 3C). MMP10 expression was significantly upregulated in ESCA, HNSC and LUSC (Fig. 3D). In addition, MMP12 was upregulated in CESC, ESCA, HNSC, LUAD, LUSC and STAD, whereas it was downregulated in pancreatic adenocarcinoma (Fig. 3E). Overall, the expression level of $M M P 1, L A M C 2, M M P 3, M M P 10$ and $M M P 12$ was significantly upregulated in HNSC tissues.

Correlations among genes expression in samples from the TCGA-HNSC database. To select the five genes of interest for further investigation, the correlations between gene expression patterns in samples from TCGA were analyzed using cBioPortal (35-36). The results demonstrated that $M M P 1$ expression level was positively correlated with LAMC2 (Fig. 4A), MMP3 (Fig. 4B) and MMP1O (Fig. 4C) expression levels. In addition, $L A M C 2$ expression level was positively correlated with MMP10 expression level (Fig. 4D).
Pearson correlation coefficients ranged from 0.33 to 0.69 , and Spearman correlation coefficients ranged from 0.55 to 0.83 . All together, these results suggested that $M M P 1$, $L A M C 2, M M P 3$ and MMP10 expression levels were positively correlated in HNSC tissues.

\section{Discussion}

The American Joint Committee on Cancer has updated the Tumor-Node-Metastasis system for cancer staging system according to diagnostic class, treatment choices and prognosis for OTSCC (37). However, with the development of novel modern therapeutic approaches for cancer, genetic analysis is an outstanding tool for early diagnosis of cancer that can prolong patient survival (38). Previous clinical studies on OTSCC development and progression reported increased incidence and mortality rates due to the lack of knowledge on 
OTSCC $(39,40)$. The present study identified 11 DEGs (five upregulated and six downregulated genes) from four mRNA expression profile datasets via GEO. These DEGs were significantly associated with OTSCC pathogenesis. The present study therefore focused on the five upregulated DEGs since they may be considered as potential reliable biomarkers. These five target genes were categorized into three classes according to their associated biological functions (molecular functions, biological processes and cellular components) thanks to the GO enrichment using multiple approaches. The results demonstrated that all genes were involved in extracellular matrix disassembly and were localized in the extracellular region during cancer development. Furthermore, expression levels of these genes were positively correlated with each other and upregulated in OTSCC and HNSC tissues compared with adjacent normal tissues, as confirmed using DAVID.

Expression levels of four MMP family members, including $M M P 1, M M P 3, M M P 10$ and MMP12, were upregulated in HNSC tissues compared with adjacent normal tissues. These genes serve important functions in numerous physiological and pathological processes involved in tumor progression and can promote tumor-induced angiogenesis and extracellular matrix disassembly, enhancing therefore tumor invasion and metastasis $(41,42) . M M P 1$ is known to be significantly associated with poor clinical outcomes and is therefore a robust prognostic factor for various types of cancer, including colorectal cancer and breast cancer (43-45). Furthermore, MMP3 has been proposed as a vital tumor oncogene in numerous cancers, including pancreatic, pulmonary, and mammary carcinoma (46). In addition, MMP10 has been demonstrated to be a potential clinical marker for cancer stem-like cells/cancer-initiating cells in epithelial ovarian cancer (EOC) and may serve as a therapeutic target in chemotherapy-resistant EOCs (47). MMP12 has been reported to be highly expressed in nasopharyngeal carcinoma both in vitro and in vivo, and is therefore a powerful tumor marker for cancer metastasis (48). Furthermore, $L A M C 2$, which encodes the major component of laminin-5, was one of the upregulated genes in the present study. Highly expression levels of LAMC2 have been previously reported in numerous invasive tumors $(49,50)$, including carcinomas of the lung $(51)$, the colorectum $(52,53)$ and the pancreas $(54,55)$. In addition, it has been reported that $L A M C 2$ overexpression in esophageal cancer is associated with poor survival $(56,57)$. Subsequently, $M M P 1, M M P 3, M M P 10$, MMP12 and LAMC2 may serve as novel promising prognostic factors in advanced metastasis of OTSCC. The protein expression of MMP1, MMP3, MMP10, MMP12 and LAMC2 should be further confirmed in OTSCC tissues. Furthermore, the correlation between MMP1, MMP3, MMP10, MMP12 and LAMC2 protein expression and the survival and clinic characteristics of patients with OTSCC should be further assessed. Tissue samples from patients with OSTCC are currently being collected for this purpose, and will be used for immunohistochemistry analysis to detect MMPs protein expression. Furthermore, the clinical significance, biological function and underlying mechanism of MMPs on the pathogenesis and progression of OTSCC will be further investigated.

In conclusion, the present study identified five upregulated genes that may be considered as robust and reliable biomarkers in OTSCC prognosis. The findings from this study may help discovering the role of oncogenes in cancer progression and determining the underlying pathways in order to develop novel therapeutic strategies for OTSCC.

\section{Acknowledgements}

Not applicable.

\section{Funding}

The present study was funded by the National Natural Science Foundation of China (grant no. 81673721) and the Natural Science Foundation of Fujian Province (grant no. 2017I0007).

\section{Availability of data and materials}

The datasets used and/or analyzed during the current study are available from the corresponding author on reasonable request.

\section{Authors' contributions}

PZ, LL, YC and JP conceived and designed the experiments. PZ, AS, ZC and XH performed GEO and GEO2R analyses. $\mathrm{LL}, \mathrm{XH}, \mathrm{YC}$ and JL conducted gene ontology analyses using DAVID. BW, JL, YC and JP analyzed TCGA database using GEPIA. LL, AS, ZC and YC analyzed expressions of genes in OTSCC from the TCGA database. XH, JL, BW and JL assessed the correlations among gene expressions using cBioPortal. PZ, LL, YC and JP drafted the manuscript.

\section{Ethics approval and consent to participate}

Not applicable.

\section{Patient consent for publication}

Not applicable.

\section{Competing interest}

The authors declare that they have no competing interests.

\section{References}

1. Jemal A, Bray F, Center MM, Ferlay J, Ward E and Forman D: Global cancer statistics. CA Cancer J Clin 61: 69-90, 2011.

2. Ledgerwood LG, Kumar D, Eterovic AK, Wick J, Chen K, Zhao H, Tazi L, Manna P, Kerley S, Joshi R, et al: The degree of intratumor mutational heterogeneity varies by primary tumor sub-site. Oncotarget 7: 27185-27198, 2016.

3. Ye H, Yu T, Temam S, Ziober BL, Wang J, Schwartz JL, Mao L, Wong DT and Zhou X: Transcriptomic dissection of tongue squamous cell carcinoma. BMC Genomics 9: 69, 2008.

4. Döbrossy L: Epidemiology of head and neck cancer: Magnitude of the problem. Cancer Metastasis Rev 24: 9-17, 2005.

5. Tímár J, Csuka O, Remenár E, Répássy G and Kásler M: Progression of head and neck squamous cell cancer. Cancer Metastasis Rev 24: 107-127, 2005.

6. Stransky N, Egloff AM, Tward AD, Kostic AD, Cibulskis K, Sivachenko A, Kryukov GV, Lawrence MS, Sougnez C, McKenna A, et al: The mutational landscape of head and neck squamous cell carcinoma. Science 333: 1157-1160, 2011.

7. Patel SC, Carpenter WR, Tyree S, Couch ME, Weissler M, Hackman T, Hayes DN, Shores C and Chera BS: Increasing incidence of oral tongue squamous cell carcinoma in young white women, age 18 to 44 years. J Clin Oncol 29: 1488-1494, 2011. 
8. Ng JH, Iyer NG, Tan MH and Edgren G: Changing epidemiology of oral squamous cell carcinoma of the tongue: A global study. Head Neck 39: 297-304, 2017.

9. Bello IO, Soini Y and Salo T: Prognostic evaluation of oral tongue cancer: Means, markers and perspectives (I). Oral Oncol 46: 630-635, 2010.

10. Almangush A, Heikkinen I, Mäkitie AA, Coletta RD, Läärä E, Leivo I and Salo T: Prognostic biomarkers for oral tongue squamous cell carcinoma: A systematic review and meta-analysis. Br J Cancer 117: 856-866, 2017.

11. van Dijk BA, Brands MT, Geurts SM, Merkx MA and Roodenburg JL: Trends in oral cavity cancer incidence, mortality, survival and treatment in the Netherlands. Int J Cancer 139: 574-583, 2016.

12. Siegel RL, Miller KD and Jemal A: Cancer statistics, 2017. CA Cancer J Clin 67: 7-30, 2017.

13. Thangaraj SV, Shyamsundar V, Krishnamurthy A, Ramani P, Ganesan K, Muthuswami M and Ramshankar V: Molecular portrait of oral tongue squamous cell carcinoma shown by integrative meta-analysis of expression profiles with validations. PLoS One 11: e0156582, 2016.

14. Ding X, Zhang N, Cai Y, Li S, Zheng C, Jin Y, Yu T, Wang A and Zhou X: Down-regulation of tumor suppressor MTUS1/ATIP is associated with enhanced proliferation, poor differentiation and poor prognosis in oral tongue squamous cell carcinoma. Mol Oncol 6: 73-80, 2012.

15. Eslami A, Miyaguchi K, Mogushi K, Watanabe H, Okada N, Shibuya H, Mizushima H, Miura M and Tanaka H: PARVB overexpression increases cell migration capability and defines high risk for endophytic growth and metastasis in tongue squamous cell carcinoma. Br J Cancer 112: 338-344, 2015.

16. Zhao T, Ding X, Chang B, Zhou X and Wang A: MTUS1/ATIP3a down- regulation is associated with enhanced migration, invasion and poor prognosis in salivary adenoid cystic carcinoma. BMC Cancer 15: 203, 2015.

17. Rogler A, Hoja S, Giedl J, Ekici AB, Wach S, Taubert H, Goebell PJ, Wullich B, Stöckle M, Lehmann J, et al: Loss of MTUS1/ATIP expression is associated with adverse outcome in advanced bladder carcinomas: Data from a retrospective study. BMC Cancer 14: 214, 2014

18. Bravou V, Antonacopoulou A, Papanikolaou S, Nikou S, Lilis I, Giannopoulou E and Kalofonos HP: Focal adhesion proteins $\alpha$ - and $\beta$-parvin are overexpressed in human colorectal cancer and correlate with tumor progression. Cancer Invest 33: 387-397, 2015.

19. Qiu Z, Sun W, Gao S, Zhou H, Tan W, Cao M and Huang W: A 16-gene signature predicting prognosis of patients with oral tongue squamous cell carcinoma. PeerJ 5: e4062, 2017.

20. Bullinger L, Döhner K, Bair E, Fröhling S, Schlenk RF, Tibshirani R, Döhner $\mathrm{H}$ and Pollack JR: Use of gene-expression profiling to identify prognostic subclasses in adult acute myeloid leukemia. N Engl J Med 350: 1605-1616, 2004.

21. Valk PJ, Verhaak RG, Beijen MA, Erpelinck CA, Barjesteh van Waalwijk van Doorn-Khosrovani S, Boer JM, Beverloo HB, Moorhouse MJ, van der Spek PJ, Löwenberg B and Delwel R: Prognostically useful gene-expression profiles in acute myeloid leukemia. N Engl J Med 350: 1617-1628, 2004.

22. Vogelstein B, Papadopoulos N, Velculescu VE, Zhou S, Diaz LA $\mathrm{Jr}$ and Kinzler KW: Cancer genome landscapes. Science 339: 1546-1558, 2013

23. Estilo CL, O-charoenrat P, Talbot S, Socci ND, Carlson DL, Ghossein R, Williams T, Yonekawa Y, Ramanathan Y, Boyle $\mathrm{JO}$, et al: Oral tongue cancer gene expression profiling: Identification of novel potential prognosticators by oligonucleotide microarray analysis. BMC Cancer 9: 11, 2009.

24. Reis PP, Waldron L, Perez-Ordonez B, Pintilie M, Galloni NN, Xuan Y, Cervigne NK, Warner GC, Makitie AA, Simpson C, et al: A gene signature in histologically normal surgical margins is predictive of oral carcinoma recurrence. BMC Cancer 11: 437, 2011.

25. Krishnan NM, Dhas K, Nair J, Palve V, Bagwan J, Siddappa G, Suresh A, Kekatpure VD, Kuriakose MA and Panda B: A minimal DNA methylation signature in oral tongue squamous cell carcinoma links altered methylation with tumor attributes. Mol Cancer Res 14: 805-819, 2016.

26. Clough $\mathrm{E}$ and Barrett T: The gene expression omnibus database. Methods Mol Biol 1418: 93-110, 2016.

27. Davis S and Meltzer PS: GEOquery: A bridge between the Gene Expression Omnibus (GEO) and BioConductor. Bioinformatics 23: 1846-1847, 2007.
28. Ashburner M, Ball CA, Blake JA, Botstein D, Butler H, Cherry JM, Davis AP, Dolinski K, Dwight SS, Eppig JT, et al: Gene ontology: Tool for the unification of biology. The Gene Ontology Consortium. Nat Genet 25: 25-29, 2000.

29. Huang da W, Sherman BT and Lempicki RA: Systematic and integrative analysis of large gene lists using DAVID bioinformatics resources. Nat Protoc 4: 44-57, 2009.

30. Mandel-Brehm C, Retallack H, Knudsen GM, Yamana A, Hajj-Ali RA, Calabrese LH, Tihan T, Sample HA, Zorn KC, Gorman MP, et al: Exploratory proteomic analysis implicates the alternative complement cascade in primary CNS vasculitis Neurology 93: e433-e444, 2019.

31. Zhu Y, Qiu P and Ji Y: TCGA-assembler: Open-source software for retrieving and processing TCGA data. Nat Methods 11: 599-600, 2014

32. Doecke JD, Chekouo TT, Stingo F and Do KA: miRNA target gene identification: Sourcing miRNA target gene relationships for the analyses of TCGA illumina MiSeq and RNA-Seq Hiseq platform data. Int J Hum Genet 14: 17-22, 2017.

33. Chen WJ, Tang RX, He RQ, Li DY, Liang L, Zeng JH, Hu XH, Ma J, Li SK and Chen G: Clinical roles of the aberrantly expressed lncRNAs in lung squamous cell carcinoma: A studybased on RNA-sequencing and microarray data mining. Oncotarget 8: 61282-61304, 2017.

34. Tang Z, Li C, Kang B, Gao G, Li C and Zhang Z: GEPIA: A web server for cancer and normal gene expression profiling and interactive analyses. Nucleic Acids Res 45: W98-W102, 2017.

35. Cerami E, Gao J, Dogrusoz U, Gross BE, Sumer SO, Aksoy BA, Jacobsen A, Byrne CJ, Heuer ML, Larsson E, et al: The cBio cancer genomics portal: An open platform for exploring multidimensional cancer genomics data. Cancer Discov 2: 401-404, 2012.

36. Gao J, Aksoy BA, Dogrusoz U, Dresdner G, Gross B, Sumer SO, Sun Y, Jacobsen A, Sinha R, Larsson E, et al: Integrative analysis of complex cancer genomics and clinical profiles using the cBioPortal. Sci Signal 6: pl1, 2013.

37. Amit M, Tam S, Takahashi H, Choi KY, Zafereo M, Bell D and Weber RS: Prognostic performance of the American Joint Committee on Cancer 8th edition of the TNM staging system in patients with early oral tongue cancer. Head Neck 41: 1270-1276, 2019.

38. Cancer Genome Atlas Network: Comprehensive genomic characterization of head and neck squamous cell carcinomas. Nature 517: 576-582, 2015.

39. Ashley EA: The precision medicine initiative: A new national effort. JAMA 313: 2119-2120, 2015.

40. Pitiyage G, Tilakaratne WM, Tavassoli Mand Warnakulasuriya S: Molecular markers in oral epithelial dysplasia: Review. J Oral Pathol Med 38: 737-752, 2009.

41. Duffy MJ: Use of biomarkers in screening for cancer. Adv Exp Med Biol 867: 27-39, 2015.

42. Kerkelä E and Saarialho-Kere U: Matrix metalloproteinases in tumor progression: Focus on basal and squamous cell skin cancer. Exp Dermatol 12: 109-125, 2003.

43. Stamenkovic I: Matrix metalloproteinases in tumor invasion and metastasis. Semin Cancer Biol 10: 415-433, 2000.

44. Li H, Zhong A, Li S, Meng X, Wang X, Xu F and Lai M: The integrated pathway of $\mathrm{TGF} / \mathrm{Sn}$ ail with $\mathrm{TNF} \alpha / \mathrm{NF} \kappa \mathrm{B}$ may facilitate the tumor-stroma interaction in the EMT process and colorectal cancer prognosis. Sci Rep 7: 4915, 2017.

45. Shen CJ, Kuo YL, Chen CC, Chen MJ and Cheng YM: MMP1 expression is activated by Slug and enhances multi-drug resistance (MDR) in breast cancer. PLoS One 12: e0174487, 2017.

46. Lu X, Wang Q, Hu G, Van Poznak C, Fleisher M, Reiss M, Massagué J and Kang Y: ADAMTS1 and MMP1 proteolytically engage EGF-like ligands in an osteolytic signaling cascade for bone metastasis. Genes Dev 23: 1882-1894, 2009.

47. Mehner C, Miller E, Nassar A, Bamlet WR, Radisky ES and Radisky DC: Tumor cell expression of MMP3 as a prognostic factor for poor survival in pancreatic, pulmonary, and mammary carcinoma. Genes Cancer 6: 480-489, 2015.

48. Mariya T, Hirohashi Y, Torigoe T, Tabuchi Y, Asano T, Saijo H, Kuroda T, Yasuda K, Mizuuchi M, Saito T and Sato N: Matrix metalloproteinase-10 regulates stemness of ovarian cancer stem-like cells by activation of canonical Wnt signaling and can be a target of chemotherapy-resistant ovarian cancer. Oncotarget 7: 26806-26822, 2016. 
49. Chung IC, Chen LC, Chung AK, Chao M, Huang HY, Hsueh C Tsang NM, Chang KP, Liang Y, Li HP and Chang YS: Matrix metalloproteinase 12 is induced by heterogeneous nuclear ribonucleoprotein $\mathrm{K}$ and promotes migration and invasion in nasopharyngeal carcinoma. BMC Cancer 14: 348, 2014.

50. Koshikawa N, Moriyama K, Takamura H, Mizushima H, Nagashima Y, Yanoma S and Miyazaki K: Overexpression of laminin gamma 2 chain monomer in invading gastric carcinoma cells. Cancer Res 59: 5596-5601, 1999.

51. Pyke C, Salo S, Ralfkiaer E, Rømer J, Danø K and Tryggvason K: Laminin-5 is a marker of invading cancer cells in some human carcinomas and is coexpressed with the receptor for urokinase plasminogen activator in budding cancer cells in colon adenocarcinomas. Cancer Res 55: 4132-4139, 1995.

52. Kagesato Y, Mizushima H, Koshikawa N, Kitamura H, Hayashi H, Ogawa N, Tsukuda M and Miyazaki K: Sole expression of laminin gamma 2 chain in invading tumor cells and its association with stromal fibrosis in lung adenocarcinomas. Jpn J Cancer Res 92: 184-192, 2001.

53. Hlubek F, Jung A, Kotzor N, Kirchner T and Brabletz T: Expression of the invasion factor laminin gamma 2 in colorectal carcinomas is regulated by beta-catenin. Cancer Res 61: 8089-8093, 2001.
54. Sordat I, Bosman FT, Dorta G, Rousselle P, Aberdam D, Blum AL and Sordat B: Differential expression of laminin-5 subunits and integrin receptors in human colorectal neoplasia. J Pathol 185: 44-52, 1998.

55. Soini Y,Määttä M, Salo S, Tryggvason K and Autio-Harmainen H: Expression of the laminin gamma 2 chain in pancreatic adenocarcinoma. J Pathol 180: 290-294, 1996.

56. Takahashi S, Hasebe T, Oda T, Sasaki S, Kinoshita T, Konishi M, Ochiai $\mathrm{T}$ and Ochiai A: Cytoplasmic expression of laminin gamma 2 chain correlates with postoperative hepatic metastasis and poor prognosis in patients with pancreatic ductal adenocarcinoma. Cancer 94: 1894-1901, 2002.

57. Yamamoto $\mathrm{H}$, Itoh $\mathrm{F}$, Iku S, Hosokawa $\mathrm{M}$ and Imai $\mathrm{K}$ : Expression of the gamma(2) chain of laminin-5 at the invasive front is associated with recurrence and poor prognosis in human esophageal squamous cell carcinoma. Clin Cancer Res 7: 896-900, 2001.

(i) $\odot$ This work is licensed under a Creative Commons Attribution-NonCommercial-NoDerivatives 4.0 International (CC BY-NC-ND 4.0) License. 